\title{
Palmoplantar Malignant Melanoma in the Brazzaville University Hospital
}

\section{Nsondé Malanda J ${ }^{\star 1}$, Kimpamboudi $\mathrm{AVM}^{2}$, Soussa $\mathrm{RB}^{3}$, Nkoua Mbon JB ${ }^{1}$ and Gombé Mbalawa $\mathrm{Ch}^{4}$}

${ }^{1}$ Carcinology and Radiotherapy department

${ }^{2}$ Surgery Department Makelekele Hospital Brazzaville

${ }^{3}$ Dermatology and Venereal disease Department

${ }^{4}$ National Institute of Health Research Brazzaville

*Corresponding author: Nsondé Malanda J, Carcinology and radiotherapy department BUH POBox 32, E-mail: drjmalandam@yahoo.fr

Citation: Nsondé Malanda J, Kimpamboudi AVM, Soussa RB, Nkoua Mbon JB, Gombé Mbalawa Ch (2017) Palmoplantar Malignant Melanoma in the Brazzaville University Hospital. J Cancer Sci Clin Oncol 4(1): 101. doi: 10.15744/2394-6520.4.101

Received Date: October 19, 2016 Accepted Date: January 27, 2017 Published Date: January 30, 2017

\begin{abstract}
Palmoplantar Malignant Melanoma (PPMM) is a tumor that arises from the melanocytes of the palmoplantar location. It is usually curable when treated surgically if diagnosed early.

Objective: To assess the clinical presentation and treatment outcome of PPMM in Brazzaville

Patients and Methods: This is a cross sectional study of thirteen years (January 1998-December 2011). 121 patients with PPMM who received the 3 treatment types were included: Surgery, chemotherapy and combination of surgery and chemotherapy. The evaluation was done at the 3rd and 6th treatment course. The analysis was done using Stata10.0. the survival was calculated using the Kaplan Meier method.

Results: We noted predominantly female (65 versus 56) were affected. The mean age was $61,20 \pm 10$, 78 years (27-90 years). The job categories affected were farmers and housewives. Pain was the main complaint to seek medical care. The mean time delay between first observation and consultation with a physician was $16,46 \pm 30,31$. The ulcero (0) and nodular form in $43,8 \%$, Clark levels were known in $50 \%$ of the patient and classified as T4 $68,5 \%$ of cases No molecular study was done in all patients. Complete response in 61 patients and the overall survival at 49 months is $60 \%$. Survival without relapse at 95 months is $50 \%$.

Conclusion: The PPMM is a rare malignant tumor, with a non-negligible frequency (occurrence) in our setting; this tumor remains (aggressive) with high metastatic potential. The large surgical removal at an early stage remains the only treatment with a curative, which is difficult to realize at a later stage when the cancer is advanced at the time of diagnosis. The public awareness campaign is important to change the people attitude and the early search for symptoms is the basis of prevention.
\end{abstract}

Keywords: Palmo plantar; Malignant Melanoma; Chemotherapy; Brazzaville

\section{Introduction}

Malignant melanoma is a tumor arises from melanocytes which are found on all sites of the body $[1,2]$. We can observe cutaneous in $90 \%$ of the cases but also in other sites such as eye mucus and meningeal [2]. In France, it represents 5\% of all cutaneous cancers [3]. The palmoplantar sites were studied because of the incidence, a low survival rate due to less technical equipment in our setting, we will describe the diagnosis and treatment characteristics.

\section{Patients and Methods}

This is a retrospective study done in the department of Carcinology and Radiotherapy of the University Hospital center of Brazzaville from 01 January 1998 to 31 December 2011. The data were collected from medical files of patients followed in the department.

\section{Inclusion Citeria}

Patients with palmoplantar malignant melanoma with:

- Demographic details (sex, age, socio-professional status)

- Localisation : palmoplantar

- Clinical description

- Microscopic diagnosis and/or Clark level 
- TNM Staging of UICC (2002)

- Information on the treatment received and followup every 3 months

\section{Exclusion Criteria}

Patients with malignant melanoma not localized in the plantar or palmar area, patients with incomplete records and with a follow-up delay of less than 3 months were excluded.

\section{Methods}

The evaluation of the general status according to the WHO performance indicators

\section{Treatments Received}

\section{Chemotherapy:}

\section{$\triangleright$ First Line}

\section{Mono Chemotherapy}

- Dacarbazine protocol (DTIC) $1000 \mathrm{mg} / \mathrm{m}^{2} / \mathrm{d}$ I.V. D1-D22

- Temozolomide protocol (TMZ) : $150 \mathrm{mg} / \mathrm{m}^{2} / \mathrm{d}$ P.O. D1-5. D1-D29

- Carmustine protocol : $100 \mathrm{mg} / \mathrm{m}^{2} /$ day, D1-2, every 6-8 weeks

\section{Polychemotherapy}

- CVD protocol : Cisplatine 20mg/m²/d IV D1-4, 22-26-Vinblastine 2mg/m²/d IV D1-J 1- 4,22-26- Dacarbazine 800mg/m²/d IV D1, 22

- Dacarbazine-cyclophosphamide protocol (CPM) : DTIC $200 \mathrm{mg} / \mathrm{m}^{2} / \mathrm{d}$ D1 to $5 \mathrm{PIV}$; CPM: $600 \mathrm{mg} / \mathrm{m}^{2} / \mathrm{d}$ D1 IV, D1-D28

- Farmorubicine (EPI)-cisplatine (CDDP) protocol: EPI $75 \mathrm{mg} / \mathrm{m}^{2} / \mathrm{d}$ D1 IV; CDDP: $100 \mathrm{mg} / \mathrm{m}^{2} / \mathrm{d}$ D1 PIV

\section{$\checkmark$ Second line}

- Carmustine-Dacarbazine-Methotrexate (MTX) : BCNU 200mg/m²/d D1 IV; DTIC 250mg/m²/d D1 and D8 PIV MTX $300 \mathrm{mg} / \mathrm{m}^{2} / \mathrm{d}$ D1 PIV

\section{Surgery}

$\checkmark$ Conservative surgery: the excision by biopsy: with a healthy margin of 1 to $3 \mathrm{~cm},+/$ - lymphadenectomy

$\rightarrow$ Radical surgery : amputation of finger, toenail, limb or of the leg $+/$ - lymphadenectomy

$\checkmark$ Wide excision : Lymph nodes and fistular and necrotizing plantar ulcerations for patients comfort.

\section{Assessment}

Treatment assessment at 3 and 6 courses of chemotherapy according to WHO criteria.

The minimum follow-up is 6 months

Data analysis: done with the method Stata 10.0 the survival was calculated by the Kaplan-Meier method and followed by the student test.

\section{Results}

\section{Epidemiological Features}

From 1998 to 2011 (13 years), 7074 new cases of cancers registered, the cancer registry of Brazzaville, noted 315 cases (4, 45\%) of skin cancer, of which 126 cases of malignant Melanoma and 121 cases located at the palmoplantar site (Table1and 2). The frequency of $1,7 \%$ of all cancer types and $34,8 \%$ compared to skin cancers with a sex ratio of $1: 16$ (Figures 1, 2, 3, 4, 5, and 6).

\begin{tabular}{|c|c|c|c|c|}
\hline $\begin{array}{c}\text { Malignant } \\
\text { Melanoma }\end{array}$ & Men & Women & Total & Percentage \% \\
\hline Cutaneous & & & & \\
\hline$\bullet$ Plantar & 55 & 65 & 120 & \\
\hline • Palmar & 01 & & 01 & \\
\hline$\bullet$ Facial & & 01 & 01 & \\
\hline Sub total & $\mathbf{5 6}$ & $\mathbf{6 6}$ & $\mathbf{1 2 2}$ & $\mathbf{9 6 , 8}$ \\
\hline Extracutaneous & & & & \\
\hline$\bullet$ Endometrial & & 01 & 01 & \\
\hline - Tongue & 01 & & 01 & \\
\hline
\end{tabular}




\begin{tabular}{|c|c|c|c|c|}
\hline $\begin{array}{c}\text { Malignant } \\
\text { Melanoma }\end{array}$ & Men & Women & Total & Percentage \% \\
\hline • Palate & 01 & & 01 & \\
\hline • Anorectal Junction & & 01 & 01 & \\
\hline Subtotal & $\mathbf{0 2}$ & $\mathbf{0 2}$ & $\mathbf{0 4}$ & $\mathbf{0 3 . 2 0}$ \\
\hline TOTAL & $\mathbf{5 8}$ & $\mathbf{6 8}$ & $\mathbf{1 2 6}$ & $\mathbf{1 0 0}$ \\
\hline
\end{tabular}

Table 1: Site distribution of registered Malignant Melanoma

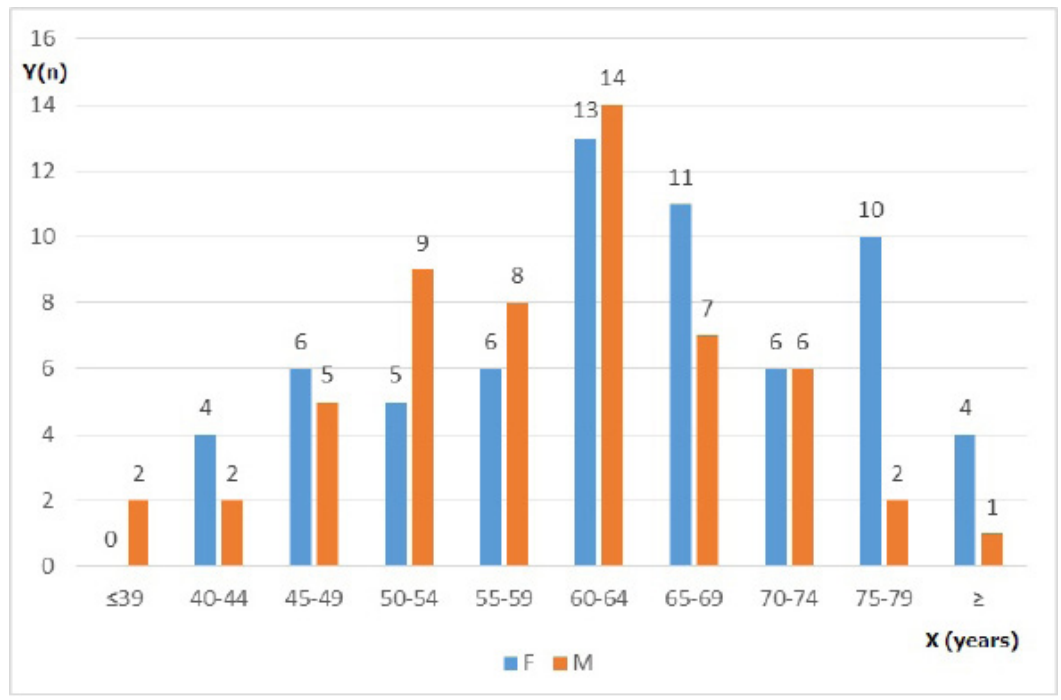

The median age is $61,20 \pm 10,78$ years, (27-90 years)

- Female $62,85 \pm 11,22$ years ( 40 and 90 years)

- Male $59.41 \pm 9,97$ years (27 and 83 years)

Figure 1: Patients distribution per age and sex

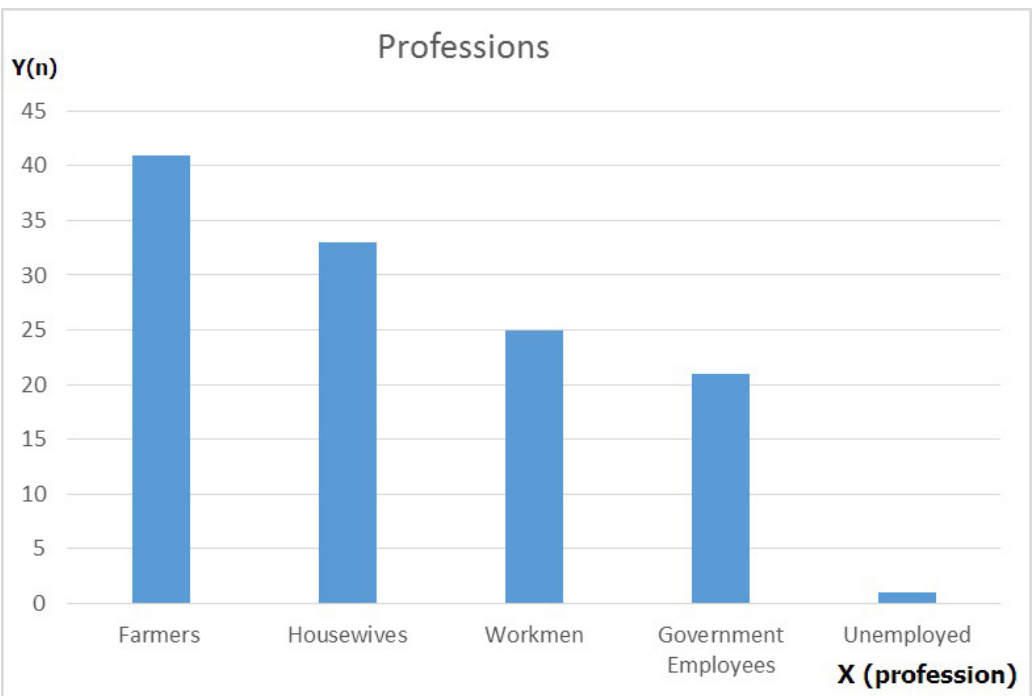

Figure 2: Patient's distribution according to profession

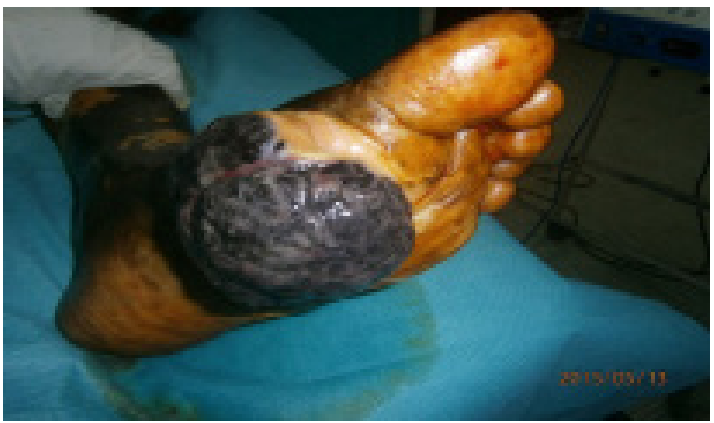

Figure 3: Black lesions of $12 \times 9 \mathrm{~cm}$ with extension to the internal margin of the foot. Patient of 54 years 


\section{Clinical features}

\begin{tabular}{|c|c|c|c|c|}
\hline Delay (months) & Men & Women & Total & Percentage \% \\
\hline $\mathbf{1 - 6}$ & 29 & 22 & 51 & 42,2 \\
\hline $\mathbf{7 - 1 2}$ & 12 & 18 & 30 & 24,8 \\
\hline $\mathbf{1 3 - 1 8}$ & 06 & 10 & 16 & 13,2 \\
\hline $\mathbf{1 9 - 2 4}$ & 02 & 06 & 08 & 6,6 \\
\hline $\mathbf{2 5 - 3 0}$ & 03 & 02 & 05 & 4,1 \\
\hline $\mathbf{3 1 - 3 6}$ & 03 & 03 & 06 & 5,0 \\
\hline $\mathbf{7 2}$ & 01 & 04 & 05 & 4,1 \\
\hline TOTAL & 56 & 65 & 121 & 100 \\
\hline
\end{tabular}

$\mathrm{P}=0,43$

The median time delay to consultation is $16,46 \pm 30,31$ months (range 1-240 months) Lesion localization: $81,7 \%(n=99)$ of the lesions were located at the sole

Table 2: Distribution according to the time delay to consultation

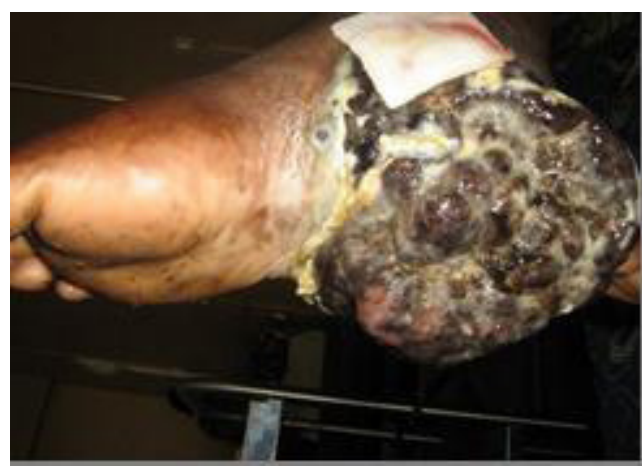

Figure 4: Black lesion of the right heel with extension at the internal malleolus in a 62 years patient

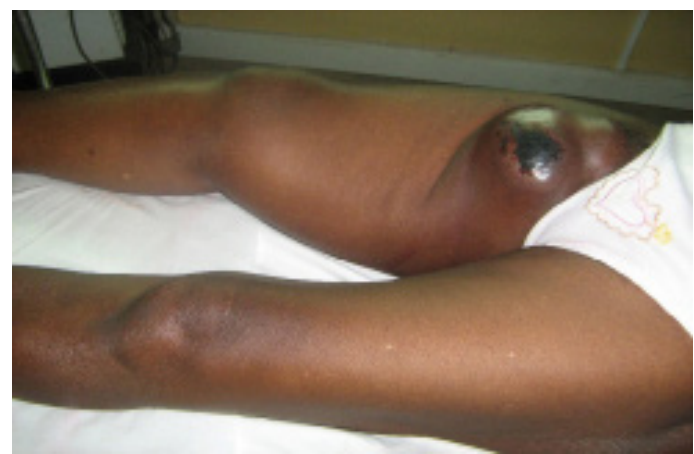

Figure 5: Voluminous right inguinal nodes in a 62 year patient with right plantar melanoma

\section{Secondary localisations}

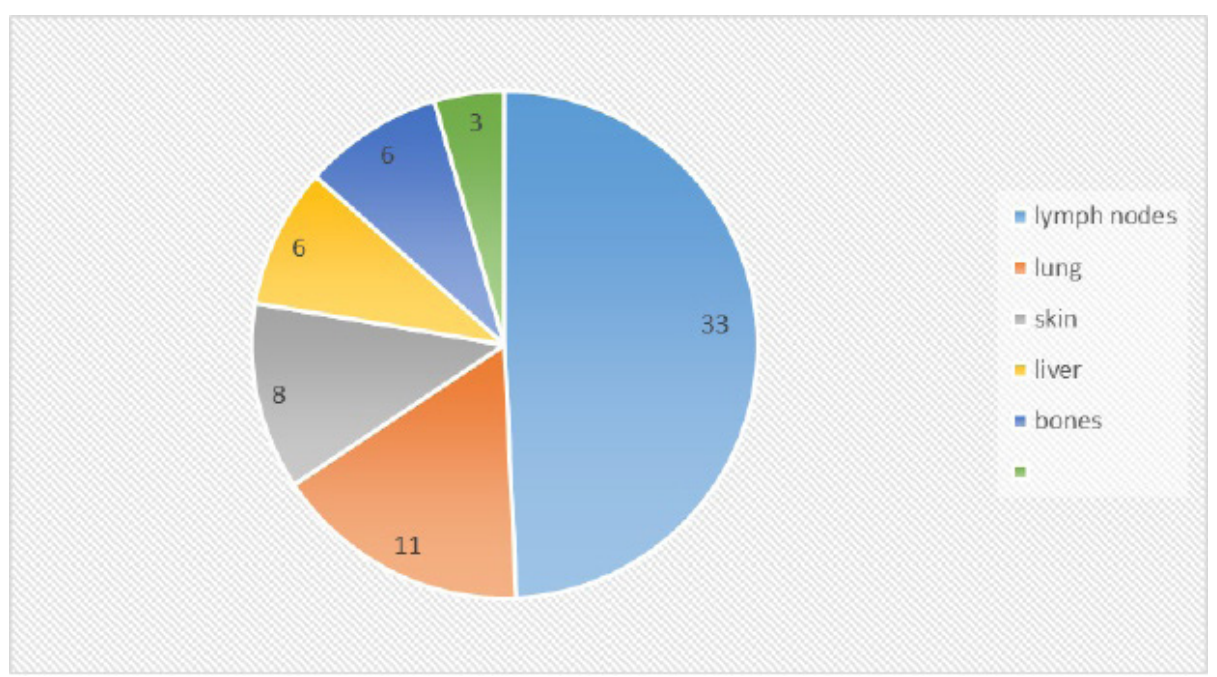

Figure 6: Metastatic localizations observed macroscopically all lesions were black 


\section{Histological types}

\begin{tabular}{|c|c|c|c|c|}
\hline Types & Male & Female & Total & Percentage \% \\
\hline Nodular MM & 17 & 13 & 30 & 24,8 \\
\hline Acrolentiginous MM & 6 & 13 & 19 & 15,7 \\
\hline Superficial spreading MM & 7 & 4 & 11 & 9,1 \\
\hline No histology indication MM & 26 & 35 & 61 & 50,4 \\
\hline TOTAL & $\mathbf{5 6}$ & $\mathbf{6 5}$ & $\mathbf{1 2 1}$ & $\mathbf{1 0 0}$ \\
\hline
\end{tabular}

Table 3: Histological types distribution

Clark and Mihn level: 85,9\% ( $\mathrm{n}=104)$ classified at Table 3, 4 and 5

\section{Patients staging}

\begin{tabular}{|c|c|c|c|c|}
\hline Stage & & Male & Female & $\begin{array}{c}\text { Total } \\
\text { (percentage \%) }\end{array}$ \\
\hline AJCC & TNM & & & \\
\hline \multicolumn{5}{|l|}{ I } \\
\hline \multirow{3}{*}{ II } & T2NM0 & 07 & 04 & 11 \\
\hline & T3NM0 & 11 & 14 & 25 \\
\hline & T4NM0 & & 02 & 02 \\
\hline Sous total & & 18 & 20 & $38(31,40)$ \\
\hline \multirow{4}{*}{ III } & T2N1M0 & 04 & 08 & 12 \\
\hline & T3N1M0 & 03 & 04 & 07 \\
\hline & T4N2M0 & 01 & 10 & 11 \\
\hline & T4N3M0 & 06 & 13 & 19 \\
\hline Sous total & & 14 & 35 & $49(40,49)$ \\
\hline \multirow{5}{*}{ IV } & T2N1M+ & 04 & & 04 \\
\hline & T3N3M+ & 02 & 03 & 05 \\
\hline & T4N1M+ & 02 & 03 & 05 \\
\hline & T4N2M+ & 06 & 02 & 08 \\
\hline & T4N3M+ & 10 & 02 & 12 \\
\hline Sous total & & 24 & 10 & $34(28,09)$ \\
\hline Total & & 56 & 65 & 121 \\
\hline
\end{tabular}

Table 4: AJCC Patient staging

Treatments (For more details on treatment check Table 5, 6, and 7)

\begin{tabular}{|c|c|c|c|c|}
\hline Therapy & Male & Female & Total & Percentage \% \\
\hline Surgery-chemotherapy & 13 & 31 & 44 & 36,4 \\
\hline Chemotherapy & 17 & 12 & 29 & 24 \\
\hline Surgery & 9 & 0 & 9 & 7,4 \\
\hline Incomplete therapy & 17 & 22 & 39 & 32,2 \\
\hline Total & $\mathbf{5 6}$ & $\mathbf{6 5}$ & $\mathbf{1 2 1}$ & $\mathbf{1 0 0}$ \\
\hline
\end{tabular}

39 patients received 1to 2 cycles of chemotherapy Table 5: Distribution according to treatment

\begin{tabular}{|c|c|c|c|c|}
\hline Surgical treatment & Site & Male & Female & Total \\
\hline Conservative & & & & \\
• Excision & Plantar & 09 & 12 & 21 \\
• Excision +lymphadenectomy & Plantar & 06 & 13 & 19 \\
\hline Radical & Toenail & 03 & 03 & 06 \\
- Amputation \pm lymphadenec- & Leg & 01 & 03 & 04 \\
tomy & Foot & 02 & 00 & 02 \\
& Thumb & 01 & 00 & 01 \\
\hline TOTAL & & 22 & 31 & 53 \\
\hline
\end{tabular}

Table 6: Distribution according to surgical treatment 


\begin{tabular}{|c|c|c|c|}
\hline $\begin{array}{c}\text { Protocols } \\
\text { (chemotherapy) }\end{array}$ & Male & Female & Total \\
\hline First line & & & \\
\hline Monochemotherapy & & 18 & 33 \\
\hline DTIC & 15 & 01 & 01 \\
\hline TMZ & 00 & 03 & 05 \\
\hline BCNU & 02 & 00 & 01 \\
\hline FTMU & 01 & & \\
\hline Polychemotherapy & & 13 & 19 \\
\hline DTIC-CPM & 06 & 06 & 10 \\
\hline DTIC-VCR-CDDP & 04 & 02 & 04 \\
\hline CDDP-EPI & 02 & & \\
\hline Second line & & 03 & 06 \\
\hline BCNU-DTIC-MTX & 03 & 03 & 05 \\
\hline DTIC-CPM & 02 & 04 & 05 \\
\hline DTIC-VCR-CDDP & 01 & 03 \\
\hline
\end{tabular}

Table 7: Distribution according to specific medical therapy

\section{Assessment}

The assessment was done in 82 patients. 42 patients benefited from surgery associated to chemotherapy, 29 received chemotherapy and 09 patients were treated only by surgery (Figures 7,8, 9 and10).

\section{Global survival curve of all patients}

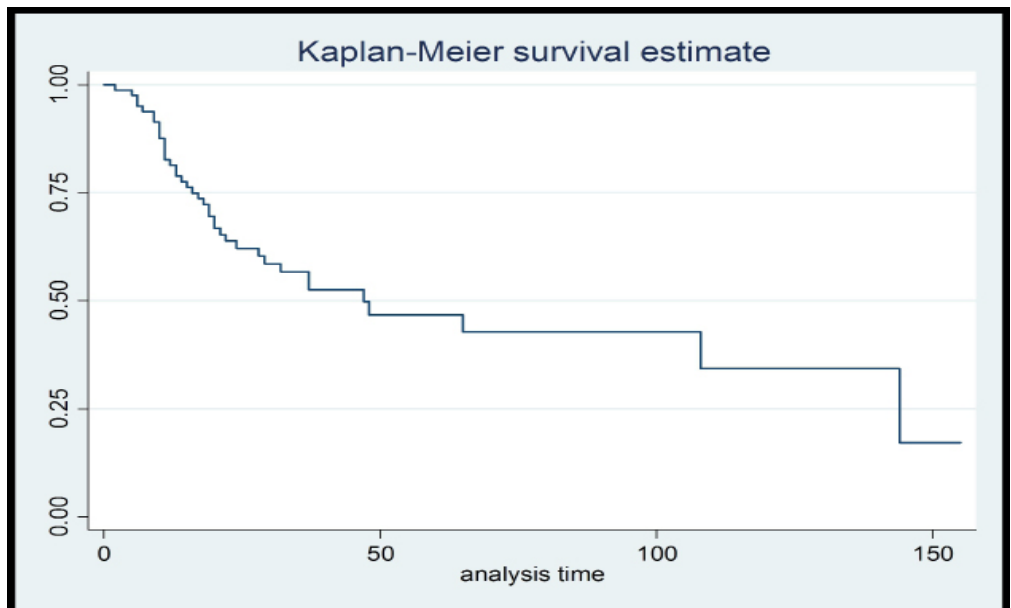

The survival rate at 49 months is $50 \%$

Figure 7: Global survival curve

Global survival according to treatment

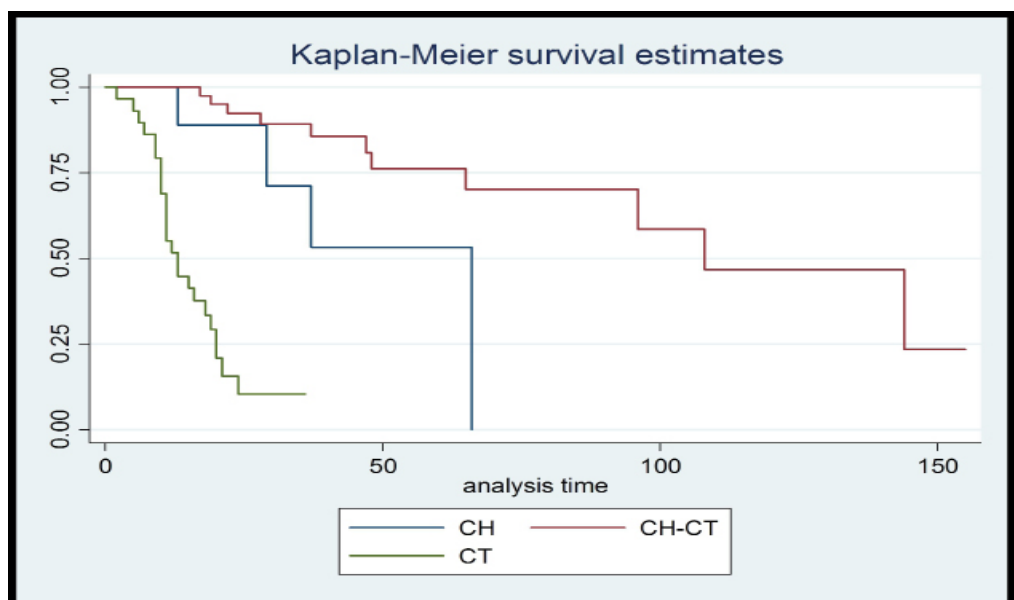

$\mathrm{P}=0,000$

The global survival rate, $50 \%$ at 13 month, 41 month and 106 month for chemotherapy, surgery and surgery-chemotherapy groups Figure 8: Global survival median according to treatment types 


\section{Survival curve without relapse}

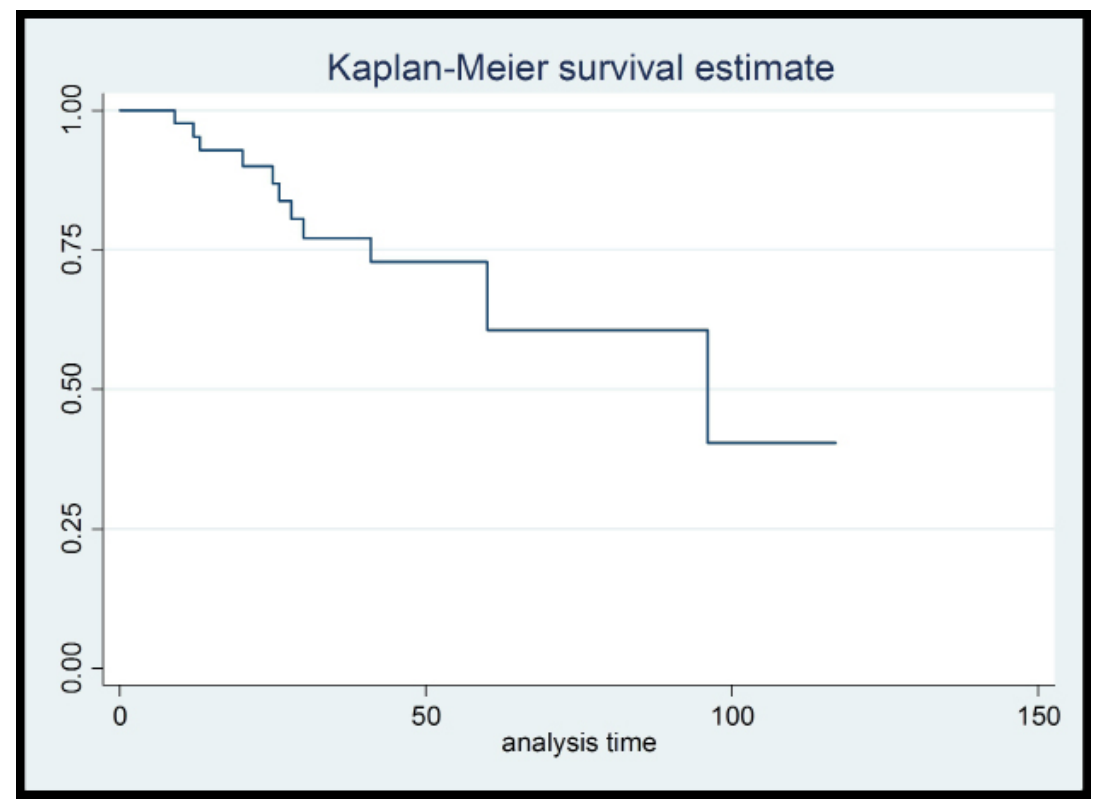

$50 \%$ of patients had a local relapse and metastases at 95 month Figure 9: Global survival without relapse

Survival without relapse according to treatment

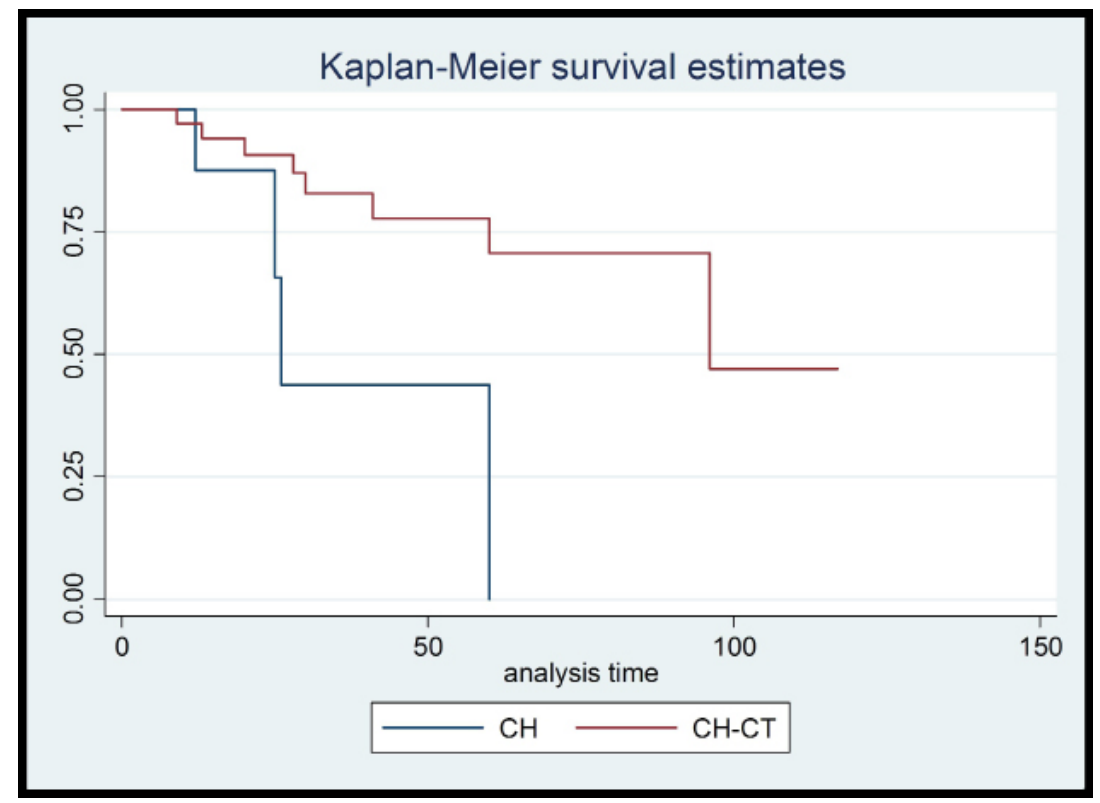

Figure 10: Survival curve according to treatment (surgery, surgery-chemotherapy)

The patients in the surgical group (50\%), with relapse at 25 month and 95 month for the surgery - chemotherapy group. No significant difference in relation to survival between those who finish their chemotherapy cycles and those who did not finished $(\mathrm{p}=0,5)$.

\section{Discussion}

The cutaneous metastatic malignant melanoma represents a frequency of less than $10 \%$ of cutaneous cancers in occident with fatal outcome. In our setting, the occurrence of PPMM is a non-negligible reality $(38,4 \%)$ in the overall skin cancers registered against $5 \%$ for other authors [2,3]. Regarding the sex ratio, the data remains contradictory, we noted a female preponderance same as, but others African authors retrieve a male preponderance [4-6]. Franke and al.[3] noted a media age of 63,3 $\pm 14,4$ years at the time of diagnosis, which is similar to the result of our population sample. We can confirm, like other authors that PPMM is a pathology of the elderly [3-5].

The palmoplantar localization of malignant melanoma, in particular at the sole of the foot is more frequent in Asian and African 
as it was noted in Husdon and al. [4] cohort with a high frequency in black subjects (75\%) than in white skin subjects (23) [6]. The female preponderance of PPMM was noted in our setting which can explain the fact that that population is professionally active, in household activities and are more exposed to micro traumas in the plantar area which was also noted by Swan and al. [7] in other risk factors that might be associated with the oncogenesis of PPMM [4]. Our results are identical as the literature review of the PPMM site preferably at the feet in the African and also in the African American and the Chinese suggesting the hypothesis of the plantar low pigmentation and micro traumas [6]. These results were identical to ours and showed that malignant melanoma on exposed skin is rare in black population (Table 1). If in white population the major risk factor appears to be sun exposure to UV [8]. We retrieve professional categories with more cases such as farmers, housewife doing housekeeping work.

In our setting nothing can explain this hypothesis, as few articles are available. Noneless the 'barefoot' notion and of traumas as part of the disease history allowed in their cohort retrieved PPMM in farmers with a tendency to walk barefoot in their farm $[9,10]$. To note that $81,7 \%$ of the cases we observed, the lesion localized in the pressure point of the extremities, sole and heel, which explain the pressure exercised at that level associated with a reduced melanin level in that region.

Franke and al. [3] estimated that 94,4\% of MM localized at the margin of the plantar pressure area. The literature review did not retrieve the plantar localization in African. The only case observed do not allow any discussion.

At the clinical level, Hudson and al. noted, at the time of diagnosis PPMM present with signs of advanced disease with ulceration, necrosis and bleeding, this was also observed in our cohort [4].

These signs explained the advanced stage of the disease at the time of first consultation; as confirmed by Francke and al. [3]. Late consultation is a frequent phenomenon in carcinology in our setting, which was demonstrated by Gombe and al. [11]. This can also be explain by the lack of national policy in the management of cancer. Hudson and al. [4] confirmed that malignant melanoma is the most aggressive skin cancers, and found 16 metastases all localized in lymph nodes. The metastases sites were predominantly localized at the sentinel nodes in our cohort, confirming that PPMM remains a lymphophilic tumor. Other sites were such as lung, liver, brain, bone and skin have been reported in publications and in our cohort $[4,9,12]$. These data is similar to what we noted in our cohort confirming the advanced stage when the patient is seen at first consultation. The fact that the recruitment of patients are done in an oncology unit is a non-negligible aspect explaining these metastatic forms.

Some African authors noted that nodular MM is first followed by the acral lentiginous [1,6,9]. Although in our study, our results were similar to those published; we cannot extrapolate our data to the reste of the cases because the histologic type was not noted in more than half of the cases.

In Africa, carcinoma are generally diagnosed at an advanced stage and metastatic [3,11]. To achieve a better prognosis of PPMM an effective early diagnosis will allow to reduce the number of advanced and metastatic forms. The prognosis is assessed by two parameters: first the Clarck level of penetration as reported by some authors [4,6\&9]. He noted that out of 195 patients, $71 \%$ cases were at 4 and 5, as in our results. In contrast the Breslow index, better prognosis indicator was not noted. In the prognosis level Francke and al.[3] noted that the plantar localization of MM was diagnosed at an advanced stage which bear a bad prognosis.

In Laforest and al [12] cohort, they noted that when ulceration was present this was a factor for bad prognosis which is now part of the TNM classification. For the same author the gender also had a prognosis value, with women had a better prognosis. We observed that the size of the lesions seen at the time of first diagnosis consultation and the nodular feature all us to conclude that PPMM had a bad prognosis in our setting.

Regarding the treatment, Lavie and al.[13] underligned that the surgical act is an essential part of the curative therapy, the biopsy should be complete to assess the level of underlying involvement. Surgery, as the only curative treatment cannot be considered in our setting due to the delay in diagnostic.

For that reasons amputations are still a possibility because of the bone involvement (phalanges, metartases, calcaeous, and malleolus). Hudson and al.[4] report that South African of black ancestry one case of amputation at the knee level for an acrolentigineous melanoma and three amputations out of 17 cases in stage 3.

Because of the lymphophilic trait of this cancer, the search for the sentinel node introduced by Morton et al. in 1992 which is recognized as a prognosis indicator could not be realized in our setting. That why curage was systematic considering the size of the lymph node in our operated patients [14]. Nowadays therapeutic options using adjuvant therapy of PPMM is in wide expansion and various options are available $[15,16]$. Hence, the discovery of molecular alterations in the melanoma had an impact in the therapeutic guidelines: the use of targeted therapy. None of our patients benefited of a genotyping nor of targeted therapy. Patients at stage IV received chemotherapy associated to radiotherapy in Hudson and al cohort [4]. Only conventional chemotherapy were used in patients at stage 4 of the disease. Non compliance and lack of medications often after 1 or 2 courses, while a minimum of 6 courses were indicated. In a study conducted by Delyon and al.[15] because of frequent chemo resistance of the metastatic malignant melanoma, the identification of genetic mutations and molecular targets had a major interest, which had a triple impact in practice: in particular change in melanoma treatment, prognosis stratification and etiological classification. Hence targeted therapy and anti-tumor immunotherapy should improve the therapeutic results in the management of melanoma. 
Regarding survival, Swan and al. [5] find a median survival of 3.92 years, which is close to the survival without relapse of our patients. The survival improvement without relapse to 3 years was significantly increased in the group treated with surgerychemotherapy combination. We are now wandering if the immunological profile of the MM of black population is the same as for other races. For patients aged more than 60 years, with PS 1or 2, the interest and feasibility of adjuvant treatment by chemotherapy based on Dacarbazine monotherapy stabilized the patients during palliative phase.

\section{Conclusion}

The palmoplantar malignant melanoma is a rare disease in our daily practice. It is an aggressive tumor with a high metastatic potential. The information, education and public communication in effort to decrease high risk behaviors and optimize early detection of potentially malignant lesions will be the basis of fighting this disease. Large excision in early stage is the only treatment with curative potential. Chemotherapy associated with surgery at stages 2 and 3 of the disease seems to improve patient survival. Molecular Study will provide indication of targeted therapies and remove survival rate.

\section{References}

1. Peko JF, Lenga Loumingou I (2003) Tumeurs malignes primitives cutanés au Congo: étude de 239 cas. Ann Univ M Ngouabi 4: 148-51.

2. Roux J, Pages C, Lebbé C (2014) Thérapies moléculaires ciblées (hors hormonothérapie). Bull Cancer. 101: S25-36.

3. Francke W, Neuman NJ, Ruzicku Schulte KM (2000) Plantar malignant melanoma: a challenge for an early recognition. Melanoma Research 10: 571-6.

4. Hudson DA, Krige Jake EJ, Stubbing H (1998) Plantar melanoma: results of treatment in three population groups. Surgery 124: 877-82.

5. Samaila MOA, Adewuyi SA (2005) A histopathological analysis of cutaneous malignancies in a tropical African population. Niger of Surg Rese 7: 300 -4.

6. Diomandé MI, Effi AB, Hondé M. D’Horpock F, Koffi K, et al. (1999) Malignant melanoma in the Ivory Coast. Epidemiologic and histo-prognostic aspects. Report of 195 cases. Clin Exp Pathol 47: 92-5.

7. Swan MC, Hudson DA (2003) Malignant melanoma in South Africans of mixed ancestry: a retrospective analysis. Melanoma Research 13: 415-9.

8. Dummer R, Hauschild A, Guggenheim M,Keilholz U, Pentheroudakis G (2012) Cutaneous melanoma: ESMO Clinical Practice Guidelines for diagnosis, treatment and followup. Ann Oncol. 23: 86-91.

9. Ngadda HA, Olasode BJ (2003) Histopathologic review of malignant melanoma in Ife-Ife, Nigeria. Niger J Med 9: 89-91.

10. Oseni GO, Olaitan PB, Komolafe AO, Olaofe OO, Akinyemi HA et al. (2015) Malignant skin lesions in Oshogbo, Nigeria. Pan Afr Med J 20: 253.

11. Gombé Mbalawa C, Doudou D, Nkoua Mbon JB, Minga B, Makouanzi Nsimba S et al. (2013) Arrivée des malades cancéreux aux stades avancés : tentative d'identification de responsabilité. Bull Cancer 100: 167-72.

12. Laforest M (2013) Le traitement pharmacologique du mélanome cutané métastatique. Pharmacothérapie 46: 87-99.

13. Lavie A, Desouches C, Casanova D, Bardot J, Grob JJ et al. (2007) Mise au point sur la prise en charge du mélanome malin cutané. Revue de la littérature. Ann Chirurg Plast Esthet 52: 1-13.

14. Morton D L, Wen DR, Wong JH, Economou JS, Cagle LA et al. (1992) Technical details of intraoperative lymphatic mapping for early stage melanoma. Arch Surg.; 127: 392-9.

15. Delyon J, Mourah S, Lebbé C (2014) Mélanomes métastatiques. In Anomalies moléculaires des cancers. Ciblage thérapeutique. K Leroy, P de Crémoux JLE 215-26.

16. Mourah S, Lebbé C (2014) Altérations moléculaires dans les mélanomes et thérapies ciblées. Bull Cancer 101: S5-11.

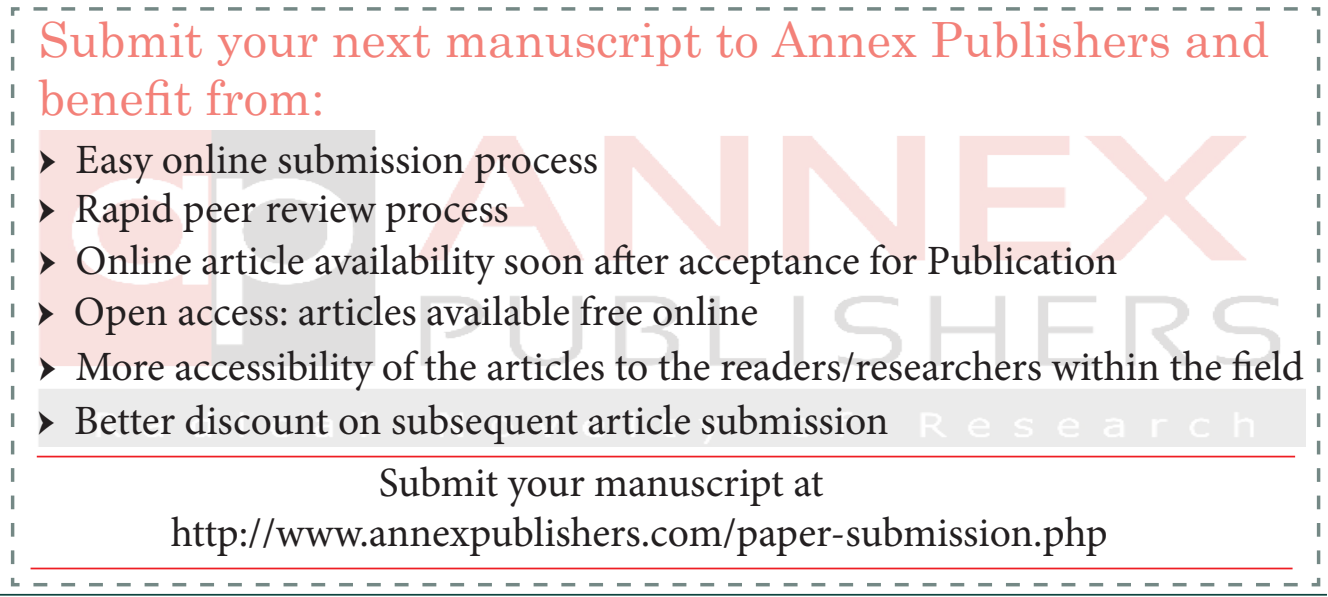

